\title{
Editorial
}

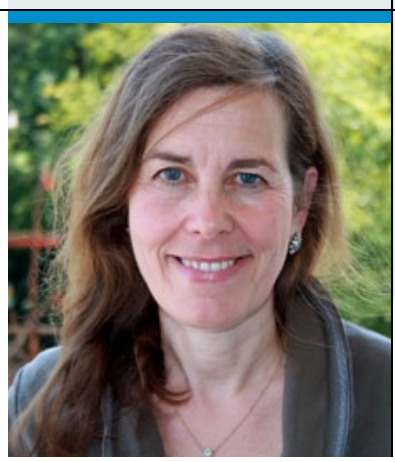

"Wir denken, dass wir wichtige, spannende und für

alle Pädiater sehr interessante Themen aus dem großen

Gebiet der Neonatologie für Sie ausgewählt haben."

Prof. Dr. med. Claudia Roll, Chefärztin der Abteilung Neonatologie und Pädiatrische Intensivmedizin, Vestische Kinder- und Jugendklinik, Universität Witten/Herdecke

\section{Neues aus der Neonatologie}

Sehr geehrte Leserinnen und Leser, liebe Kolleginnen und Kollegen,

ich freue mich, Ihnen das Sonderheft Neonatologie vorzustellen, welches pünktlich zum gemeinsamen Kongress der Gesellschaft für Neonatologie und Pädiatrische Intensivmedizin (GNPI) und der Deutschen Gesellschaft für Kinderheilkunde (DKGJ) in Hamburg im September 2012 erscheint.

pädiatrie hautnah - da nimmt die Haut des Neugeborenen natürlich einen wichtigen Platz ein. Was ist harmlos, was nicht, was erfordert weitere Diagnostik oder Therapie? Ulrich Mutschler aus Hildesheim widmet sich in seinem umfassenden Beitrag und mit eindrucksvollem Bildmaterial dem Thema.

Was sagen die aktuellen internationalen Empfehlungen zur Erstversorgung und ggf. Reanimation von Früh- und Reifgeborenen direkt nach der Geburt im Kreißsaal? Thomas Höhn aus Düsseldorf referiert und kommentiert für Sie die wichtigsten Aspekte der aktuellen Empfehlungen.

Was passiert nach einer Asphyxie im Kreißsaal, wenn sich das Neugeborene nicht prompt erholt, sondern Zeichen einer hypoxisch-ischämischen Enzephalopathie entwickelt? Christoph Bührer aus Berlin berichtet über die Datenlage und die praktischen Aspekte der therapeutischen Hypothermie.

Katrin Mehler und Bernhard Roth aus Köln stellen vor, welche Auswirkungen Schmerzen bei Neugeborenen haben, wie wir sie messen können, welche therapeutischen Optionen es gibt und wie Schmerzen vermieden werden.

Welche Kinder sollten zu Hause mit einem Monitor überwacht werden? Christian Poets aus Tübingen beschäftigt sich seit langem mit diesem Thema. Sein Beitrag legt dar, wann und wie eine Monitorüberwachung zu Hause sinnvoll ist.
Norbert Teig aus Bochum zeigt, welche Probleme Frühgeborene mit bronchopulmonaler Dysplasie nach Entlassung aus der Klinik haben können, und was therapeutisch notwendig und sinnvoll ist.

Ultraschall und MRT in der Neonatologie: Wann ist der Einsatz welcher Methode sinnvoll? Im gemeinsamen Beitrag mit Sandra Horsch nehmen wir dazu Stellung und geben praktische Ratschläge für die MRT-Durchführung.

Nachdem der Fokus lange Zeit auf der Sorge um die Langzeitentwicklung extrem unreifer Frühgeborener lag, denen der letzte Beitrag in diesem Heft gehört, hat sich in den letzten Jahren das Augenmerk auf die viel größere Zahl sogenannter später Frühgeborener gerichtet. Die Besonderheiten und Bedürfnisse dieses Kollektivs werden von Wolfram Burkhardt, Jürgen Dinger und Mario Rüdiger aus Dresden vorgestellt.

Wir denken, dass wir wichtige, spannende und für alle Pädiater sehr interessante Themen aus dem großen Gebiet der Neonatologie für Sie ausgewählt haben und hoffen, dass Ihnen die Beiträge gefallen und Sie von Ihnen profitieren können.

Ihre

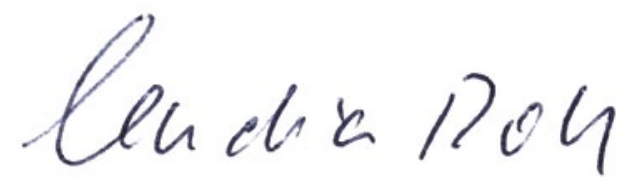

